\title{
$Z \gamma$ production in association with two jets at next-to-leading order QCD
}

\author{
Francisco Campanario $^{1, \mathrm{a}}$, Matthias Kerner ${ }^{2, \mathrm{~b}}$, Le Duc Ninh ${ }^{2, \mathrm{c}}$, Dieter Zeppenfeld ${ }^{2, \mathrm{~d}}$ \\ ${ }^{1}$ Theory Division, IFIC, University of Valencia-CSIC, 46980 Paterna, Valencia, Spain \\ ${ }^{2}$ Institute for Theoretical Physics, KIT, 76128 Karlsruhe, Germany
}

Received: 25 August 2014 / Accepted: 15 September 2014 / Published online: 25 September 2014

(C) The Author(s) 2014. This article is published with open access at Springerlink.com

\begin{abstract}
Next-to-leading order QCD corrections to the QCD-induced $p p \rightarrow l^{+} l^{-} \gamma j j+X$ and $p p \rightarrow \bar{v}_{l} \nu_{l} \gamma j j+X$ processes are presented. The latter is used to find an optimal cut to reduce the contribution of radiative photon emission off the charged leptons in the first channel. As expected, the scale uncertainties are significantly reduced at NLO and the QCD corrections are phase-space dependent and important for precise measurements at the LHC.
\end{abstract}

\section{Introduction}

The production of a prompt photon in association with two charged leptons and two jets at the LHC is an attractive mechanism to study weak boson scattering, namely $W^{+} W^{-} \rightarrow$ $\gamma V$ with $V=Z / \gamma^{*}$. It is also relevant to the study of anomalous gauge boson couplings, which may provide hints of new physics beyond the Standard Model.

At leading order (LO), the process $p p \rightarrow j j \gamma l^{+} l^{-}+$ $X$ is classified into two mechanisms: the electroweakinduced mechanism of order $\mathcal{O}\left(\alpha^{5}\right)$, which is sensitive to $W^{+} W^{-} \rightarrow \gamma V$ scattering and the QCD-induced channel of order $\mathcal{O}\left(\alpha_{s}^{2} \alpha^{3}\right)$, which can be considered as a background. The EW contributions can be further classified into $t$-channel vector-boson fusion contributions known at NLO QCD [1] and other contributions including notably tri-boson production processes with one boson decaying hadronically. The NLO QCD corrections to tri-boson production with leptonic decays were computed in $[2,3]$ and the hadronic decay modes are available via the VBFNLO program [4-6]. The interference effects between these contributions are expected to be negligible for most measurements at the LHC [7].

\footnotetext{
a e-mail: francisco.campanario@ific.uv.es

b e-mail: matthias.kerner@kit.edu

c e-mail: duc.le@kit.edu

de-mail: dieter.zeppenfeld@kit.edu
}

In this paper, we consider the QCD-induced mechanism for the processes

$$
\begin{aligned}
& p p \rightarrow l^{+} l^{-} \gamma j j+X, \quad “ Z_{l} \gamma j j ” \\
& p p \rightarrow \bar{v}_{l} \nu_{l} \gamma j j+X, \quad " Z_{\nu} \gamma j j "
\end{aligned}
$$

and will present the first theoretical prediction at NLO QCD accuracy. ${ }^{1}$ Some representative Feynman diagrams at $\mathrm{LO}$ are displayed in Fig. 1. Since the dominant contribution comes from the phase-space region where the intermediate $Z$ boson is resonant, the above processes are usually referred to as $Z_{l} \gamma j j$ and $Z_{\nu} \gamma j j$ production, accounting for the chargedlepton and neutrino pair production processes, respectively. With this result, all the QCD-induced $V V j j$ production processes are known at NLO QCD [7,9-18].

The signature of an isolated photon together with two jets and missing energy is difficult to study in experiment but is, as will be shown later, useful in a Monte Carlo analysis to find (by comparing the two processes) an optimal cut on the invariant mass of the two-charged lepton and photon system to remove the radiative QED contribution (where the photon is emitted off the final charged leptons). This contribution is unwanted because it reduces sensitivity to the weak boson scattering. The focus of this paper is therefore on process (1), however, a comparison of normalized distributions to process (2) will be performed.

We have implemented the QCD-induced processes (1) and (2) within the VBFNLO framework [4-6], a parton-level Monte Carlo program which allows the definition of general acceptance cuts and distributions. As customary in VBFNLO, all off-shell effects, virtual photon contributions and spincorrelation effects are fully taken into account. Our code will be included in the next release of VBFNLO.

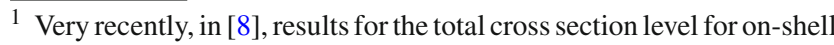
$Z \gamma j j$ production have been reported.
} 

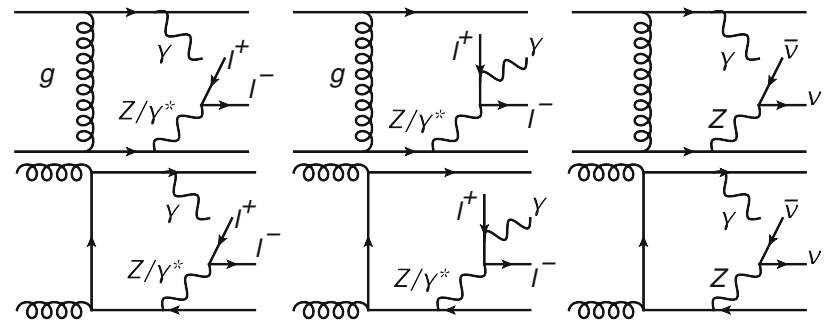

Fig. 1 Representative tree-level Feynman diagrams

In the next section we sketch the calculational setup and in Sect. 3 we define our physical observables with a set of cuts and present numerical results for the total cross section as well as various kinematical distributions. Conclusions are presented in Sect. 4. Values of the virtual amplitudes at a random phase-space point are provided in the appendix to facilitate future comparisons with our results.

\section{Calculational setup}

The calculational method of the present paper follows closely the one presented in [18] for the process $p p \rightarrow$ $l_{1}^{+} l_{1}^{-} l_{2}^{+} l_{2}^{-} j j+X$ (called from now on $Z Z j j$ for simplicity). As explained there, the gauge invariant class of closed-quark loop diagrams with EW gauge bosons directly attached to the loop are discarded. This contribution is at the few per mille level, hence negligible for all phenomenological purposes. The diagrams with a closed-quark loop and two or three gluons attached to it are, however, included. We work in the fiveflavor scheme and virtual top loops are taken into account. We use the Frixione isolation criteria [19] for the photon and therefore photon fragmentation functions are not included.

Technically, the code for the $Z_{x} \gamma j j$ processes is adapted from the $Z Z j j$ code with some modifications. This is possible because we use the effective current approach and the spinor-helicity formalism $[20,21]$ factorizing the leptonic tensor containing the EW information of the system from the QCD amplitude. For the $l_{1}^{+} l_{1}^{-} l_{2}^{+} l_{2}^{-} j j$ case, the generic amplitudes for $V_{1} V_{2} j j$ with $V_{i}=Z / \gamma^{*}(i=1,2)$ and $\hat{V} j j$ with $\hat{V}=Z / \gamma^{*}$ are first created. Then the leptonic decays $V_{i} \rightarrow l_{i}^{+} l_{i}^{-}$and $\hat{V} \rightarrow l_{1}^{+} l_{1}^{-} l_{2}^{+} l_{2}^{-}$are incorporated via effective currents. In this way, all off-shell effects and spin correlations are fully taken into account. This approach also makes it straightforward to obtain the $l^{+} l^{-} \gamma j j$ and $\bar{v}_{l} v_{l} \gamma j j$ final states by picking the relevant generic amplitudes and changing the effective currents, namely, only the $Z \gamma j j$ generic amplitude and $Z \rightarrow \bar{v}_{l} v_{l}$ effective current are needed for the neutrino channel. For the charged-lepton case, we use the $V_{1} \gamma j j$ and $\hat{V}^{\prime} j j$ generic amplitudes with $\hat{V}^{\prime} \rightarrow l^{+} l^{-} \gamma$ effective current. These trivial changes are universal and have been cross-checked. Additionally, the phase-space generator has to be modified for a fast convergence of the Monte-Carlo integration. For this purpose, it is important to notice that, for on-shell photon production, there are two contributions dominating in two different phase-space regions associated with the two decay modes of the $Z$ bosons, namely $Z \rightarrow l^{+} l^{-}$ and $Z \rightarrow l^{+} l^{-} \gamma$. This means that there are two different positions of the on-shell $Z$ pole in the phase space for the process (1). For efficient Monte Carlo generation, we divide the phase space into two separate regions to consider these two possibilities and then sum the two integrals to get the total result. The regions are generated as double EW boson production as well as $Z$ production with (approximately) onshell $Z \rightarrow l^{+} l^{-} \gamma$ three-body decay, respectively, and they are chosen according to whether $m\left(l^{+} l^{-} \gamma\right)$ or $m\left(l^{+} l^{-}\right)$is closer to $M_{Z}$. The virtual photon contribution, which is far off-shell, does not pose additional problems and is always calculated together with the corresponding $Z$ contribution. Another nontrivial change arises in the virtual amplitudes where we have to calculate a new set of scalar integrals which do not occur in the off-shell photon case. We have again checked this with two independent calculations (as explained in [18]) and obtained full agreement at the amplitude level. Further details of our calculation and implementation and checks can be found in [18]. Furthermore, we have cross-checked the LO and real emission contributions without subtraction term against Sherpa [22,23] and agreement at the per mille level was found for integrated cross sections.

With this method, we obtain the NLO inclusive cross section with statistical error of $1 \%$ in $4 \mathrm{~h}$ on an Intel $i 7-3970 X$ computer with one core and using the compiler Intel-ifort version 12.1.0. The distributions shown below are based on multiprocessor runs with a total statistical error of $0.03 \%$ at NLO.

\section{Phenomenological results}

For the numerical evaluation of the processes at the LHC operating at $14 \mathrm{TeV}$ center-of-mass energy, we use the MSTW2008 parton distribution function [24] with $\alpha_{s}^{\mathrm{LO}(\mathrm{NLO})}$ $\left(M_{Z}\right)=0.13939(0.12018)$ and the anti- $k_{\mathrm{T}}$ cluster algorithm with a cone radius of $R=0.4$. We consider jets with transverse momenta $p_{\mathrm{T}, j}>20 \mathrm{GeV}$ and rapidity $\left|y_{j}\right|<4.5$. To simulate experimental detector capabilities, we require hard and central charged leptons with $p_{\mathrm{T}, l}>20 \mathrm{GeV}$ and $\left|y_{l}\right|<2.5$ and photons with $p_{\mathrm{T}, \gamma}>30 \mathrm{GeV}$ and $\left|y_{\gamma}\right|<2.5$. We impose minimal separation distances of $R_{j l}>0.4$, $R_{l l}>0.4, R_{l \gamma}>0.4$ and $R_{j \gamma}>0.7$. To avoid the need of including photon fragmentation functions, we use the photon isolation criteria à la Frixione [19] with a cone radius of $\delta_{0}=0.7$. Events are accepted if ${ }^{2}$

\footnotetext{
2 In the 2013 Les Houches "tight isolation accord" [25], to get a lower bound of the total cross section, tighter cuts are proposed for a comparison with experimental data analyzed with the standard cone isolation criterion.
} 


$$
\sum_{i \in \text { partons }} p_{\mathrm{T}, i} \theta\left(R-R_{\gamma i}\right) \leq p_{\mathrm{T}, \gamma} \frac{1-\cos R}{1-\cos \delta_{0}} \quad \forall R<\delta_{0} .
$$

For the neutrinos of the " $Z_{v} \gamma j j$ " channel, we do not apply any cut.

Other input parameters are chosen as $M_{Z}=91.1876 \mathrm{GeV}$, $M_{W}=80.385 \mathrm{GeV}$ and $G_{F}=1.16637 \times 10^{-5} \mathrm{GeV}^{-2}$.

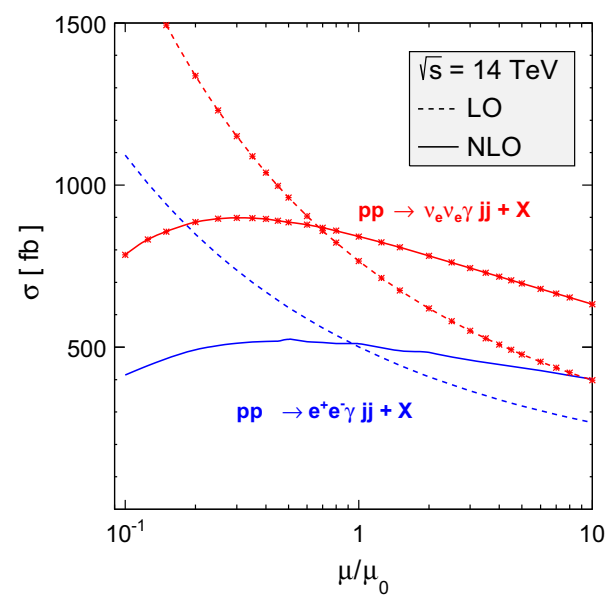

Fig. 2 Scale dependence of the total LHC cross section at LO and NLO for $p p \rightarrow e^{+} e^{-} \gamma j j+X$ and $p p \rightarrow \nu_{e} \bar{\nu}_{e} \gamma j j+X$ production around the central scale $\mu_{0}$ defined in Eq. (4). The cuts used are described in the text

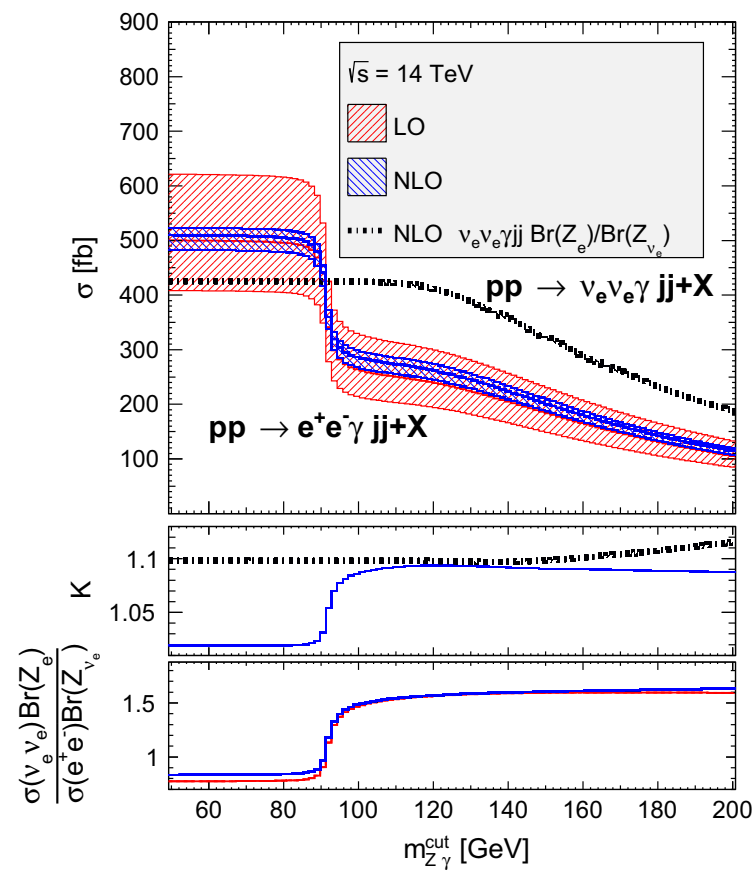

Fig. 3 Left cross section for different values of the reconstructed $Z \gamma$ invariant mass cut. The neutrino curve is multiplied by the ratio of the charge-lepton versus neutrino branching ratios. The middle panel shows the $K$-factor and the lower the ratios of the modified neutrino cross section versus the LO and NLO electron cross sections. Right normalized
The electromagnetic coupling constant and the weak-mixing angle are calculated via tree-level relations. All fermions are taken to be massless, except the top quark with $m_{\mathrm{t}}=$ $173.1 \mathrm{GeV}$. The width of the $Z$ is fixed at $\Gamma_{Z}=2.508905 \mathrm{GeV}$. The strong coupling constant is renormalized using the $\overline{\mathrm{MS}}$ scheme. The top-quark contribution is decoupled from the running, but is explicitly included in the one-loop amplitude. As a central factorization and renormalization scale, we use the sum of the transverse energy $E_{\mathrm{T}}=\left(p_{\mathrm{T}}^{2}+p^{2}\right)^{1 / 2}$ of the two tagging jets and of the reconstructed $Z \gamma$ system,

$\mu_{F}=\mu_{R}=\mu_{0}=\frac{1}{2}\left[E_{\mathrm{T}}(j j)+E_{\mathrm{T}}(V V)\right]$.

The first term interpolates between $m_{j j}$ and $\sum p_{\mathrm{T}, \mathrm{jets}}$ for large and small $\Delta y_{j j}$ values, characterizing the dynamics of these processes appropriately.

In the following, we present results for the first generation of leptons. Taking into account both the electron and the muon yields an extra factor of 2 . Summing over three generations of neutrinos gives a factor of 3 .

To evaluate the scale uncertainties associated to a fixed order calculation, we plot in Fig. 2 the cross section for the " $Z_{v} \gamma j j$ " and " $Z_{l} \gamma j j$ " channels varying the central scale in the range $\mu \in\left(10^{-1}, 10\right) \mu_{0}$ simultaneously for

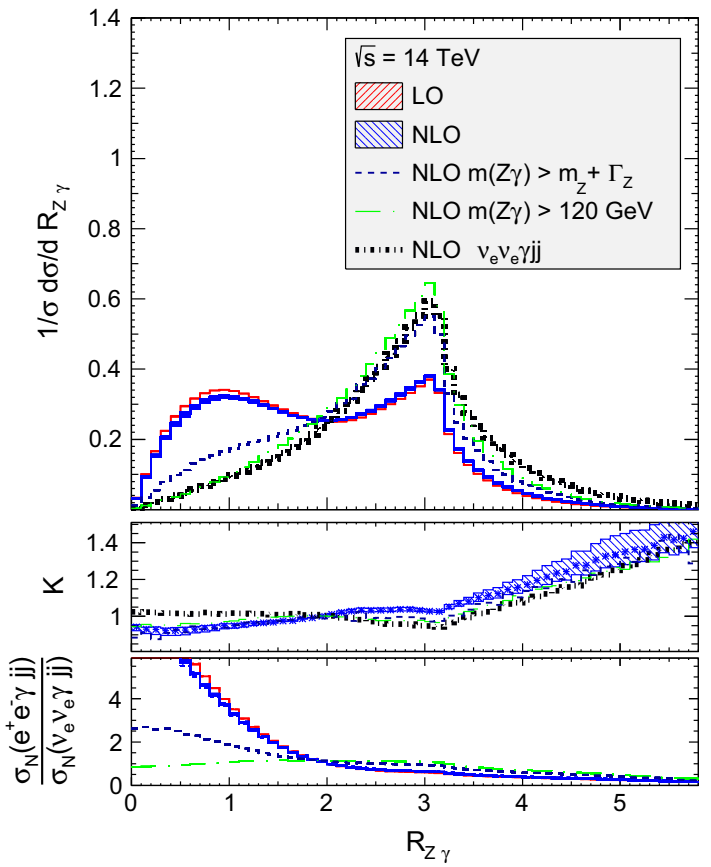

differential distributions of the rapidity-azimuthal angle separation $R_{Z \gamma}$ for different values of the $m_{Z \gamma}^{\text {cut }}$ cut. The middle and lower panels show the differential $K$-factor plots and the ratios of the normalized electron versus neutrino pair production channels 

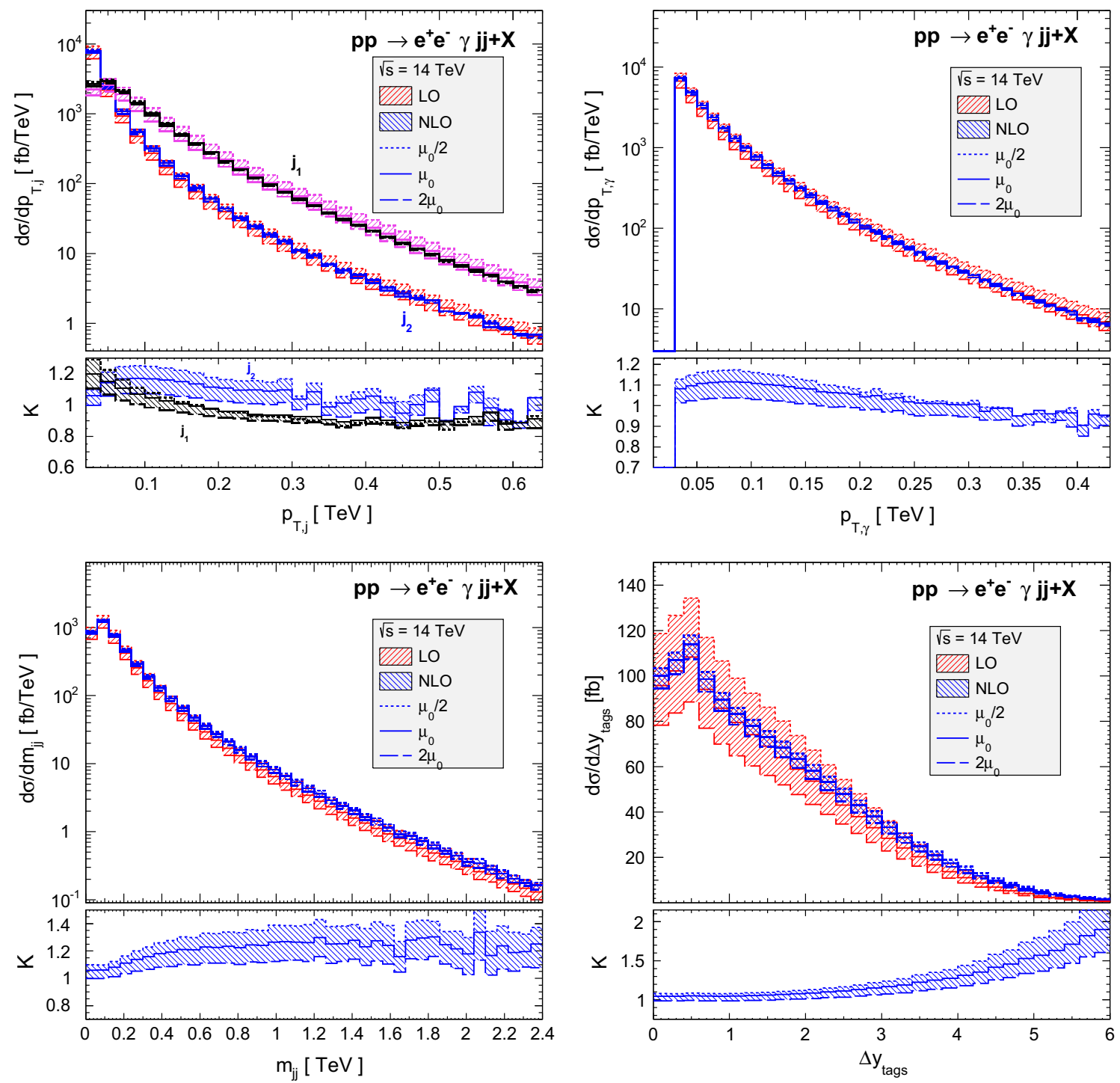

Fig. 4 Differential distributions for the transverse momentum of the tagging jets (top left) and the photon (top right). On the bottom, the invariant mass (left) and rapidity separation (right) of the two tagging jets are displayed. The bands show the scale variations around the central scale, $\mu_{0} / 2 \leq \mu_{F}=\mu_{R} \leq 2 \mu_{0}$. In the small panels, the differ-

ential $K$-factors are plotted. The bands reflect the NLO scale variations with respect to $\sigma_{\mathrm{LO}}\left(\mu_{0}\right)$. The inclusive cuts described in the text are used together with the cut on the invariant mass of the $Z \gamma$ system, $m_{Z \gamma}^{\text {cut }}=120 \mathrm{GeV}$, which eliminates the final radiative emission off the charged leptons

the factorization and the renormalization scale, which are set equal for simplicity. At the central scale, we obtain $\sigma_{\mathrm{LO}}=500.82(3)_{-18 \%}^{+24 \%} \mathrm{fb}$ and $\sigma_{\mathrm{NLO}}=510.6(1)_{-5.3 \%}^{+2.6 \%} \mathrm{fb}$ for the $e^{+} e^{-} \gamma j j$ channel and $\sigma_{\mathrm{LO}}=765.58(3)_{-19 \%}^{+26 \%} \mathrm{fb}$ and $\sigma_{\mathrm{NLO}}=840.8(3)_{-7 \%}^{+5.3 \%}$ fb for the $\bar{v}_{e} v_{e} \gamma j j$ one. The upper and lower numbers correspond to the scale uncertainties in percentage for variations of a factor 2 around the central scale and the number in brackets is the Monte-Carlo statistical error. At the central scale, we observed very mild $K$-factors, defined as the ratio of the NLO over LO predictions of the order of 1.01 and 1.1 for the " $Z_{l} \gamma j j$ " and " $Z_{\nu} \gamma j j$ " channels, respectively.

Next we investigate the radiative photon emission off the charged leptons in the " $Z_{l} \gamma j j$ " channel (see the middle Feynman diagrams of Fig. 1). These radiative decays present in both the EW- and the QCD-induced processes reduce the sensitivity to anomalous-coupling searches and therefore it is desirable to suppress them. This contribution dominates in the phase-space region where the reconstructed invariant mass of the $Z \gamma$ system is close to the $Z$ mass. Thus, imposing a cut on $M_{Z \gamma}$ around the $Z$ mass should remove this contribution. The optimum value of the cut is a priori uncertain. We therefore use the " $Z_{v} \gamma j j$ " channel, where radiative decays are absent, to determine it. 
In the left panel of Fig. 3, as functions of the $m_{Z \gamma}$ cut, we plot the integrated NLO cross sections for the " $Z_{l} \gamma j j$ " and " $Z_{v} \gamma j j$ " channels, the latter being multiplied by the ratio of the charge-lepton versus neutrino branching ratios of the $Z, \operatorname{Br}\left(Z \rightarrow e^{+} e^{-}\right) / \operatorname{Br}\left(Z \rightarrow \bar{v}_{e} v_{e}\right)=0.506$. The LO cross section is also shown for the " $Z_{l} \gamma j j$ " channel. In the bottom panel, the ratios of the modified neutrino cross sections to the LO and NLO electron cross sections are plotted. They do not converge to one in the tails due to the different cuts applied for the charged leptons and the neutrinos. As expected, for the charged-lepton case, one observes that the cross section sharply decreases when the cut value is greater than the $Z$ mass. In the middle panel, the $K$ factors are plotted. We observe that $m_{Z \gamma}^{\text {cut }}=120 \mathrm{GeV}$ is a good value since the $K$-factor exhibits a plateau and the slope of the cross section curves are approximately equal for both processes beyond this value (see bottom panel). This is confirmed in the right panel of Fig. 3, where the normalized differential distributions of the reconstructed rapidityazimuthal angle separation of the $Z \gamma$ system are plotted for the two channels. One observes that the cut $m_{Z \gamma}>m_{Z}+\Gamma_{Z}$ reduces considerably the effect of the radiative decay in the charged-lepton channel, but some remnant is still clearly visible by comparing to the neutrino channel. Increasing the cut value to $120 \mathrm{GeV}$ makes the NLO distribution of the " $Z_{l} \gamma j j$ " channel very similar to the corresponding " $Z_{v} \gamma j j$ " one. This is better seen in the bottom panel, where the ratios of the normalized differential distributions between the two channels are plotted. The ratio of the $m_{Z \gamma}^{\text {cut }}=120 \mathrm{GeV}$ curve versus the " $Z_{v} \gamma j j$ " distribution is rather flat and close to one till $R_{Z \gamma}$ reaches values of around 3 and then decreases. This difference is probably again due to the different cuts applied between the charged leptons and the neutrinos.

In the following, we impose an additional cut $m_{Z \gamma}>$ $120 \mathrm{GeV}$ and plot some relevant differential distributions for the two tagging jets and the photon at LO and NLO in the large panels of Fig. 4. The tagging jets are defined as the two jets with highest transverse momenta and are ordered by hardness. The bands show the scale uncertainty in the range $\mu_{0} / 2 \leq \mu_{F}=\mu_{R} \leq 2 \mu_{0}$. The small panels always show the differential $K$-factors where the bands represents the scale variations of the NLO result, with respect to $\sigma_{\mathrm{LO}}\left(\mu_{0}\right)$. In the top row, the differential distributions of the transverse momentum of the two tagging jets (left) and the photon (right) are plotted. The bottom row displays the invariant mass (left) and the rapidity difference (right) of the two tagging jets. As expected, the scale uncertainty decreases considerably at NLO. Note that the rapidity-separation distribution receives large NLO QCD corrections in the region selected for vector boson fusion scattering, $\Delta y_{\text {tags }}>3$. In general, the size of the $K$-factors range from 0.8 to 1.9 , showing that NLO predictions are necessary for accurate measurements.

\section{Conclusions}

In this article, we have presented first results at NLO in QCD for the $p p \rightarrow l^{+} l^{-} \gamma j j+X$ and $p p \rightarrow \bar{v}_{l} \nu_{l} \gamma j j+X$ processes. With this result, all the QCD-induced $V V j j$ production processes are known at NLO QCD.

By comparing against the neutrino production process, we have been able to efficiently remove the contribution of radiative photon emission off the charged leptons, which diminishes the sensitivity of EW-induced processes to anomalous couplings. As expected, the scale uncertainty is significantly reduced at NLO, which is visible both at the total and differential cross section level. The size of the NLO QCD corrections are phase-space dependent ranging from -20 to $+90 \%$, and they are particularly large in the region where the vectorboson scattering signal is enhanced. NLO corrections are therefore needed for reliable predictions.

Acknowledgments FC acknowledges financial support by the IEFMarie Curie program (PIEF-GA-2011-298960) and partial funding by the LHCPhenonet (PITN-GA-2010-264564) and by the MINECO (FPA2011-23596). MK is funded by the graduate program GRK 1694: "Elementary particle physics at highest energy and precision". LDN and DZ are supported in part by the Deutsche Forschungsgemeinschaft via the Sonderforschungsbereich/Transregio SFB/TR-9 "Computational Particle Physics".

Open Access This article is distributed under the terms of the Creative Commons Attribution License which permits any use, distribution, and reproduction in any medium, provided the original author(s) and the source are credited.

Funded by $\mathrm{SCOAP}^{3}$ / License Version CC BY 4.0.

\section{Appendix: Results at one phase-space point}

In the following, we present results at one phase-space point for the virtual amplitudes to facilitate comparison with future calculations. We chose the same phase-space momentum configuration as [16], Table 1, and give results for the process $j_{1} j_{2} \rightarrow j_{3} j_{4} e^{+} e^{-} \gamma$ at the squared-amplitude level, averaging over the initial-state helicities and colors.

We include all UV counterterms and all closed-quark loops with gluons attached to it. Diagrams including a closedquark loop with the $Z / \gamma^{*}$ directly attached to it are excluded. The top quark is decoupled from the running of $\alpha_{s}$. However, its contribution is explicitly included in the one-loop amplitudes. Here we use $\alpha=\alpha_{s}=1$ for simplicity. With this setup and measuring energies in $\mathrm{GeV}$, we get at tree level

$$
\begin{aligned}
& {\overline{\mid \mathcal{A}_{\mathrm{LO}}^{u u \rightarrow u u}}}^{2}=2.583569915405990 \times 10^{-2}, \\
& {\overline{\mid \mathcal{A}_{\mathrm{LO}}^{u c \rightarrow u c}}}^{2}=3.760248173799574 \times 10^{-2} \text {, } \\
& {\overline{\mid \mathcal{A}_{\mathrm{LO}}^{u d \rightarrow u d}}}^{2}=6.975514915738625 \times 10^{-2} \text {, } \\
& {\overline{\mid \mathcal{A}_{\mathrm{LO}}^{d d \rightarrow d d}}}^{2}=8.065869053590906 \times 10^{-3} \text {, }
\end{aligned}
$$


Table 1 Momenta (in $\mathrm{GeV}$ ) at a random phase-space point for $j_{1} j_{2} \rightarrow j_{3} j_{4} e^{+} e^{-} \gamma$ subprocesses
Table 2 QCD interference amplitudes $2 \operatorname{Re}\left(\mathcal{A}_{\mathrm{NLO}} \mathcal{A}_{\mathrm{LO}}^{*}\right)$ for $j_{1} j_{2} \rightarrow j_{3} j_{4} e^{+} e^{-} \gamma$ subprocesses

\begin{tabular}{lllll}
\hline & $E$ & $p_{x}$ & $p_{y}$ & $p_{z}$ \\
\hline$j_{1}$ & 32.0772251055223 & 0.0 & 0.0 & 32.0772251055223 \\
$j_{2}$ & 2801.69305619768 & 0.0 & 0.0 & -2801.69305619768 \\
$j_{3}$ & 226.525314156010 & -10.2177083492279 & $-1.251308382450315 \times 10^{-15}$ & -226.294755550298 \\
$j_{4}$ & 327.281588297290 & -6.48554750244653 & -10.1061447270513 & -327.061219882068 \\
$e^{+}$ & 646.824307052136 & 36.0746355875450 & -26.0379256562231 & -645.292438579767 \\
$e^{-}$ & 1598.85193997112 & -2.88431497177613 & 24.4490976584709 & -1598.66239347157 \\
$\gamma$ & 34.2871318266438 & -16.4870647640944 & 11.6949727248035 & 27.6949763915464 \\
\hline
\end{tabular}

\begin{tabular}{|c|c|c|c|}
\hline & $1 / \epsilon^{2}$ & $1 / \epsilon$ & Finite \\
\hline \multicolumn{4}{|l|}{$u и \rightarrow и и$} \\
\hline$I$ operator & $2.1930022552 \times 10^{-2}$ & $3.6933147142 \times 10^{-2}$ & $7.311094745 \times 10^{-2}$ \\
\hline Loop & $-2.19300225 \times 10^{-2}$ & $-3.6933147 \times 10^{-2}$ & 0.14424709 \\
\hline$I+$ loop & $3.6 \times 10^{-14}$ & $5.9 \times 10^{-13}$ & 0.2173580 \\
\hline \multicolumn{4}{|l|}{$u c \rightarrow u c$} \\
\hline$I$ operator & $3.1917977819 \times 10^{-2}$ & $5.1904760292 \times 10^{-2}$ & 0.1098476201 \\
\hline Loop & $-3.19179778 \times 10^{-2}$ & $-5.19047603 \times 10^{-2}$ & 0.196364213 \\
\hline$I+$ loop & $9.5 \times 10^{-14}$ & $1.1 \times 10^{-12}$ & 0.30621183 \\
\hline \multicolumn{4}{|l|}{$u d \rightarrow u d$} \\
\hline$I$ operator & $5.9210009570 \times 10^{-2}$ & $9.6286844080 \times 10^{-2}$ & 0.2037747651 \\
\hline Loop & $-5.92100095 \times 10^{-2}$ & $-9.628684 \times 10^{-2}$ & 0.53005178 \\
\hline$I+$ loop & $3.0 \times 10^{-13}$ & $1.4 \times 10^{-9}$ & 0.7338265 \\
\hline \multicolumn{4}{|l|}{$d d \rightarrow d d$} \\
\hline$I$ operator & $6.8465222944 \times 10^{-3}$ & $1.1487721719 \times 10^{-2}$ & $2.246881141 \times 10^{-2}$ \\
\hline Loop & $-6.84652229 \times 10^{-3}$ & $-1.14877217 \times 10^{-2}$ & $4.789599960 \times 10^{-2}$ \\
\hline$I+$ loop & $2.5 \times 10^{-14}$ & $2.5 \times 10^{-13}$ & $7.03648110 \times 10^{-2}$ \\
\hline \multicolumn{4}{|l|}{$d s \rightarrow d s$} \\
\hline$I$ operator & $1.04197858244 \times 10^{-2}$ & $1.6944572384 \times 10^{-2}$ & $3.586031267 \times 10^{-2}$ \\
\hline Loop & $-1.041978582 \times 10^{-2}$ & $-1.69445724 \times 10^{-2}$ & $6.930377376 \times 10^{-2}$ \\
\hline$I+$ loop & $5.6 \times 10^{-14}$ & $4.9 \times 10^{-13}$ & 0.105164086 \\
\hline \multicolumn{4}{|l|}{$g g \rightarrow \bar{u} u$} \\
\hline$I$ operator & $4.2224900184 \times 10^{-4}$ & $2.5147277683 \times 10^{-5}$ & $5.73018157 \times 10^{-4}$ \\
\hline Loop & $-4.2224900 \times 10^{-4}$ & $-2.51472 \times 10^{-5}$ & $1.24085333 \times 10^{-3}$ \\
\hline$I+$ loop & $2.6 \times 10^{-14}$ & $2.4 \times 10^{-13}$ & $1.81387148 \times 10^{-3}$ \\
\hline \multicolumn{4}{|l|}{$g g \rightarrow \bar{d} d$} \\
\hline$I$ operator & $1.13509295313 \times 10^{-4}$ & $6.3611573393 \times 10^{-6}$ & $1.56909560 \times 10^{-4}$ \\
\hline Loop & $-1.135092953 \times 10^{-4}$ & $-6.36116 \times 10^{-6}$ & $3.27276628 \times 10^{-4}$ \\
\hline$I+$ loop & $5.4 \times 10^{-16}$ & $2.4 \times 10^{-14}$ & $4.8418618 \times 10^{-4}$ \\
\hline
\end{tabular}

$$
\begin{aligned}
& {\overline{\mid \mathcal{A}_{\mathrm{LO}}^{d s \rightarrow d s}}}^{2}=1.227552097429276 \times 10^{-2}, \\
& {\overline{\mid \mathcal{A}_{\mathrm{LO}}^{g g \rightarrow \bar{u} u}}}^{2} \mid=3.061233143517198 \times 10^{-4}, \\
& {\overline{\mid \mathcal{A}_{\mathrm{LO}}^{g g \rightarrow \bar{d} d}}}^{2} \mid=8.229230037027499 \times 10^{-5} .
\end{aligned}
$$

For the one-loop integrals, we use the convention

$$
T_{0}=\frac{\mu_{R}^{2 \epsilon} \Gamma(1-\epsilon)}{i \pi^{2-\epsilon}} \int d^{D} q \frac{1}{\left(q^{2}-m_{1}^{2}+i 0\right) \ldots},
$$


with $D=4-2 \epsilon$. Additionally, the conventional dimensional regularization method [26] with $\mu_{R}=M_{Z}$ is used. With this, the interference amplitudes $2 \operatorname{Re}\left(\mathcal{A}_{\mathrm{NLO}} \mathcal{A}_{\mathrm{LO}}^{*}\right)$, for the one-loop corrections and the $I$-operator contribution as defined in [27], are given in Table 2.

Switching from the conventional dimensional regularization to dimensional reduction method induces a finite shift, which can be calculated noting that the sum $\left|\mathcal{A}_{\mathrm{LO}}\right|^{2}+$ $2 \operatorname{Re}\left(\mathcal{A}_{\mathrm{NLO}} \mathcal{A}_{\mathrm{LO}}^{*}\right)$ should be constant [28]. The shift on the Born amplitude squared comes from the change in the strong coupling constant, see e.g. [29],

$\alpha_{s}^{\overline{\mathrm{DR}}}=\alpha_{s}^{\overline{\mathrm{MS}}}\left(1+\frac{\alpha_{s}}{4 \pi}\right)$.

Finally, using the rule given in [27], the shift on the $I$-operator contribution can be calculated.

\section{References}

1. J. Bernabeu, F. Campanario, J. Parra (in preparation)

2. G. Bozzi, F. Campanario, V. Hankele, D. Zeppenfeld, Phys. Rev. D 81, 094030 (2010). arXiv:0911.0438

3. G. Bozzi, F. Campanario, M. Rauch, H. Rzehak, D. Zeppenfeld, Phys. Lett. B 696, 380 (2011). arXiv:1011.2206

4. K. Arnold et al., Comput. Phys. Commun. 180, 1661 (2009). arXiv:0811.4559

5. K. Arnold et al. (2011). arXiv: 1107.4038

6. J. Baglio et al. (2014). arXiv:1404.3940

7. F. Campanario, M. Kerner, L.D. Ninh, D. Zeppenfeld, Phys. Rev. D 89, 054009 (2014). arXiv: 1311.6738
8. J. Alwall et al. (2014). arXiv:1405.0301

9. T. Melia, K. Melnikov, R. Rontsch, G. Zanderighi, JHEP 1012, 053 (2010). arXiv:1007.5313

10. T. Melia, K. Melnikov, R. Rontsch, G. Zanderighi, Phys. Rev. D 83, 114043 (2011). arXiv:1104.2327

11. N. Greiner et al., Phys. Lett. B 713, 277 (2012). arXiv:1202.6004

12. A. Denner, L. Hosekova, S. Kallweit, Phys. Rev. D 86, 114014 (2012). arXiv:1209.2389

13. F. Campanario, M. Kerner, L.D. Ninh, D. Zeppenfeld, Phys. Rev. Lett. 111, 052003 (2013). arXiv:1305.1623

14. T. Gehrmann, N. Greiner, G. Heinrich, Phys. Rev. Lett. 111, 222002 (2013). arXiv: 1308.3660

15. S. Badger, A. Guffanti, V. Yundin, JHEP 1403, 122 (2014). arXiv: 1312.5927

16. F. Campanario, M. Kerner, L.D. Ninh, D. Zeppenfeld (2014). arXiv: 1402.0505

17. Z. Bern et al. (2014). arXiv:1402.4127

18. F. Campanario, M. Kerner, L.D. Ninh, D. Zeppenfeld (2014). arXiv: 1405.3972

19. S. Frixione, Phys. Lett. B 429, 369 (1998). hep-ph/9801442

20. K. Hagiwara, D. Zeppenfeld, Nucl. Phys. B 313, 560 (1989)

21. F. Campanario, JHEP 1110, 070 (2011). arXiv: 1105.0920

22. T. Gleisberg et al., JHEP 0902, 007 (2009). arXiv:0811.4622

23. T. Gleisberg, S. Hoeche, JHEP 0812, 039 (2008). arXiv:0808.3674

24. A. Martin, W. Stirling, R. Thorne, G. Watt, Eur. Phys. J. C 63, 189 (2009). arXiv:0901.0002

25. J. Butterworth et al. (2014). arXiv:1405.1067

26. G. 't Hooft, M. Veltman, Nucl. Phys. B 44, 189 (1972)

27. S. Catani, M. Seymour, Nucl. Phys. B 485, 291 (1997). hep-ph/9605323

28. S. Catani, M. Seymour, Z. Trocsanyi, Phys. Rev. D 55, 6819 (1997). hep-ph/9610553

29. Z. Kunszt, A. Signer, Z. Trocsanyi, Nucl. Phys. B 411, 397 (1994). hep-ph/9305239 